\title{
A cooperative transponder system for improved traffic safety, localizing road users in the $5 \mathrm{GHz}$ band
}

\author{
B. Schaffer, G. Kalverkamp, M. Chaabane, and E. M. Biebl \\ Fachgebiet Höchstfrequenztechnik, Technische Universität München, Munich, Germany \\ Correspondence to: B. Schaffer (schaffer@tum.de)
}

\begin{abstract}
We present a multi-user cooperative mobile transponder system which enables cars to localize pedestrians, bicyclists and other road users in order to improve traffic safety. The system operates at a center frequency of $5.768 \mathrm{GHz}$, offering the ability to test precision localization technology at frequencies close to the newly designated automotive safety related bands around $5.9 \mathrm{GHz}$. By carrying out a roundtrip time of flight measurement, the sensor can determine the distance from the onboard localization unit of a car to a road user who is equipped with an active transponder, employing the idea of a secondary radar and pulse compression. The onboard unit sends out a pseudo noise coded interrogation pulse, which is answered by one or more transponders after a short waiting time. Each transponder uses a different waiting time in order to allow for time division multiple access. We present the system setup as well as range measurement results, achieving an accuracy up to centimeters for the distance measurement and a range in the order of hundred meters. We also discuss the effect of clock drift and offset on distance accuracy for different waiting times and show how the system can be improved to further increase precision in a multiuser environment.
\end{abstract}

\section{Introduction}

Each year, thousands of pedestrians are killed in traffic accidents: in German statistics alone, 3648 fatalities are reported for the year 2010 (Statistisches Bundesamt Deutschland, 2010). Therefore, one goal of the research initiative "Ko-FAS" is to find ways to lower this number by assisting drivers in recognizing and avoiding critical traffic situations. In the project "Ko-TAG", which is a part of the research initiative Ko-FAS, a new cooperative transponder system is developed. The system is able to detect and localize pedestrians, bicyclists and other vulnerable road users even if they are hidden behind obstacles so that the driver cannot see them. Based on the localization data of the road users around the ego-vehicle and its own driving status, the onboard computer can set up risk models for every user and can prevent dangerous situations by either warning the driver or applying autonomous maneuvers (Schwarz et al., 2011). A first approach to a cooperative sensor system for pedestrian detection and localization was developed in the AMULETT project (Morhart and Biebl, 2009). The AMULETT system can determine the range to a transponder with a standard deviation of less than $10 \mathrm{~cm}$, using an RF-bandwidth of $60 \mathrm{MHz}$ and a center frequency of $2.44 \mathrm{GHz}$. Signal processing is done by an FPGA in real-time, thus an update rate of more than $50 \mathrm{~Hz}$ can be accomplished. Since the $2.4 \mathrm{GHz}$ ISM Band is already heavily used by wireless LAN, Bluetooth and other unlicensed applications, and due to the latest designations of separate frequency bands in the 5.8 to $5.9 \mathrm{GHz}$ range for automotive safety and intelligent transport systems (Electronic Communications Committee, 2008), current localization technology must be tested at these new frequencies. Therefore, we designed an improved system, based on the AMULETT technology. This new system, called "SafeTAG 1.0" is considerably smaller, consumes less power and for the first time can demonstrate multi-user detection and localization with 20 units available. Originally, the SafeTAG 1.0 system operates at $2.44 \mathrm{GHz}$, but the frequency of operation can be moved to $5.768 \mathrm{GHz}$ by connecting a frequency converter module.

\section{Theory of operation}

Many different approaches to detect and localize pedestrians in traffic scenarios have been proposed in the past, including radar, lidar and image processing via cameras. An overview over the use of these technologies is given in Gandhi and Trivedi (2007). The use of active transponders allows for simultaneous detection, classification and localization of 
pedestrians, other cars and transponder-equipped landmarks in real-time and with high accuracy, but at the cost of a more complex cooperative system. In the field of cooperative distance measurement systems, multiple realizations have been shown in the past. A ranging system based on frequency modulated continous wave chirps is presented in Roehr et al. (2008), for example. The system proposed in this work is based on the well known principle of pulse compression by pseudo noise codes. Since the basics of the distance measurement are already discussed in detail in Morhart and Biebl (2009), only a short overview of the principle of operation shall be given here.

A measurement cycle begins with the transmission of an interrogation pulse from the onboard-unit. The pulse consists of a short continuous wave preamble and a 256 bit binary maximum-length sequence, which modulates the carrier signal by on-off-keying. The bandwidth of the pseudo noise code is approximately $60 \mathrm{MHz}$. Once a transponder receives the interrogation pulse and detects its presence, a wait timer is started with a preset delay time. After the waiting time, the signal is sent back to the onboard unit, using the amplified original received samples to avoid jitter due to clock phase offset between the onboard unit and the transponder. The sign of the received signal is thereby inverted to make interrogation pulses and transponder responses distinguishable. This ensures that a second transponder does not erroneously respond to the answer of the first transponder. Each transponder has a different tag ID and therefore uses a different waiting time, defined as

$t_{\text {wait }}=5 \mu \mathrm{s}+(\operatorname{tag} \mathrm{ID}-1) \cdot 100 \mu \mathrm{s}$,

so that every answer to the interrogation pulse can be sent in a different time slot, enabling the system to support many users by a time division multiple access scheme. For every time slot, the onboard unit calculates the signal roundtrip time of flight by correlating the transmitted sequence with the received signal, taking advantage of the special correlation characteristics of the used m-sequences (Lüke, 1992). From the interpolated maximum of the correlation result, the signal roundtrip time of flight $t_{\text {RTOF }}$ and therefore the distance $R$ to a transponder can be calculated using its assigned delay time $t_{\text {wait }}$ and the speed of light $c_{0}$ :

$R=\frac{c_{0}}{2} \cdot\left(t_{\mathrm{RTOF}}-t_{\mathrm{wait}}\right)$

At the same time, a trigger signal is issued to a direction-ofarrival unit, which then estimates the angle to each transponder by using the buffered receive signals of a 6-element antenna array and a super-resolution algorithm. To ease the direction-of-arrival estimation, the transponders append a $65 \mu$ s long continuous wave burst to the end of the answered interrogation pulse. The distance measurement module and the direction-of-arrival estimation module send their localization data to a central onboard computer, which sets up the environment model and reacts to critical traffic scenarios by either warning the driver or applying autonomous avoidance or mitigation maneuvers (Geissler et al., 2011).

\section{Hardware setup}

The SafeTAG 1.0 system is based on the AMULETT technology, but the transceiver has been redesigned and optimized. The operation frequency of the base system is still $2.44 \mathrm{GHz}$, but it can be converted to $5.768 \mathrm{GHz}$ by using a frequency converter unit, offering the ability to test the localization technology at both bands. The SafeTAG 1.0 base system provides separate transmit- and receive connectors, whereas the commercial converter used for changing the center frequency only offers a single $5 \mathrm{GHz}$ and a single $2.4 \mathrm{GHz}$ connector, switching the direction of the conversion internally after autosensing the input power at the $2.4 \mathrm{GHz}$ port. Therefore, it is necessary to implement a TX/RX switch between the SafeTAG and the converter unit. This switch is controlled by the internal TX/RX enable signals of the FPGA which also switch the TX and RX components on and off. Since the converter unit is attached to the power amplifier output of the base system, a $6 \mathrm{~dB}$ attenuation pad has to be inserted into the $2.44 \mathrm{GHz}$ transmit path to avoid overloading the converter input and to set the output power of the $5 \mathrm{GHz}$ system to $22 \mathrm{dBm}$. Figures 1 and 2 show a simplified block diagram and a photograph of the transceiver and converter unit.

\section{Measurements}

In the following section, laboratory and real-world measurements will be shown in order to analyze the performance of the transponder system at the new operating frequency of $5.768 \mathrm{GHz}$. The main reasons for distance measurement errors will be identified by example measurements and ideas to improve the system performance will be given.

\subsection{Calibration}

The distance between an onboard unit and a transponder causes a time delay in the roundtrip time of flight, which is measured by the onboard unit. But components in the transceivers like filters, amplifiers and transmission lines add additional delay to the signal, making calibration necessary before useful measurements can be carried out. Calibration is achieved by measuring distance at an arbitrary position, and then determining the offset between the mean displayed and the true distance. This offset is then subtracted from all following measurements, leaving possibilities for errors only due to clock noise and drift, signal noise, and effects of multipath propagation. The severity of these effects will be discussed in the following section, and example values from measurements will be given. 

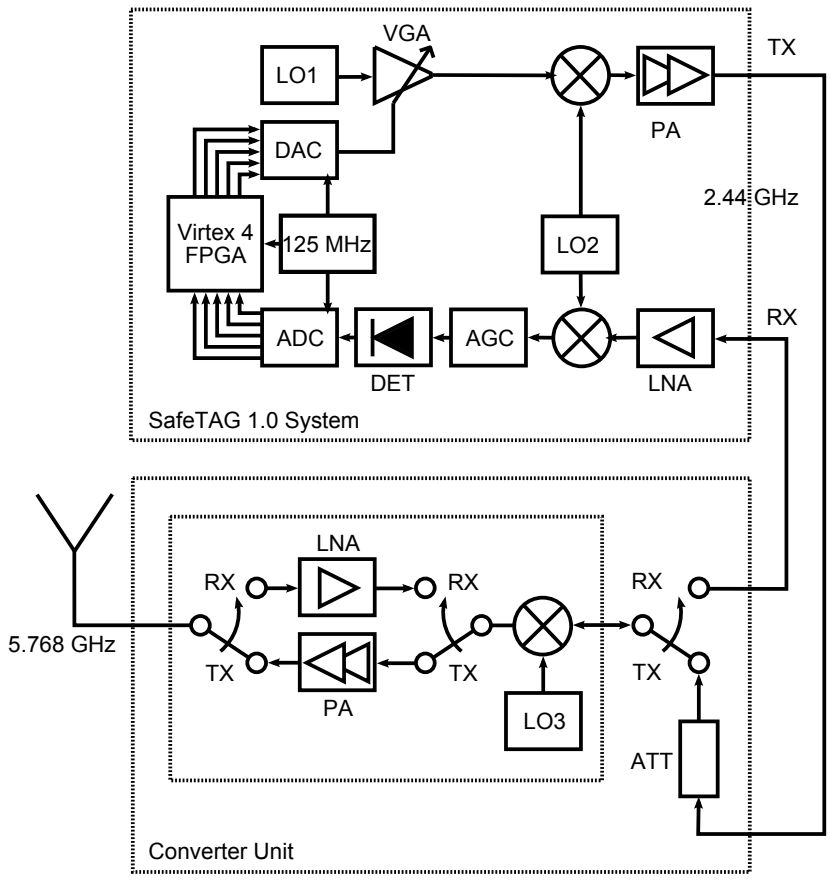

Fig. 1. Simplified block diagram of the transponder system.

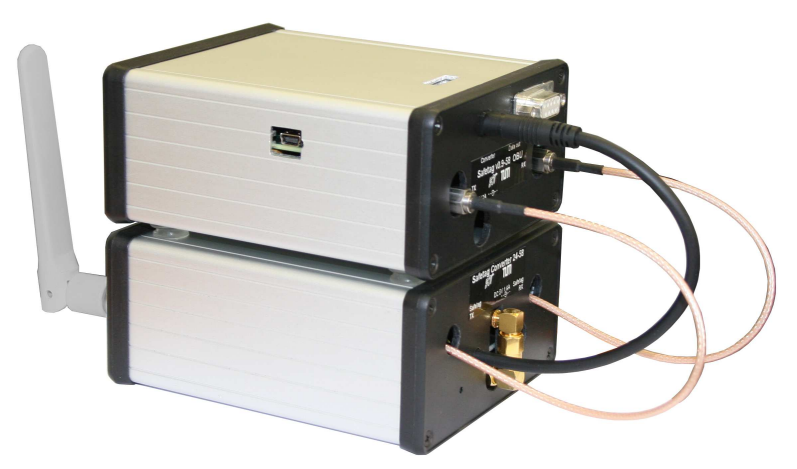

Fig. 2. Photograph of a transponder (top) with the converter unit (bottom) and a $5 \mathrm{GHz}$ antenna attached.

\subsection{Impact of clock phase and signal noise}

Phase noise and short term changes in reference oscillator frequency as well as signal noise can affect the quality of the distance measurement, even directly after calibration. The standard deviation over many acquired samples at a static distance relative to the calibration distance can be used as an indicator for the error. For comparison, the results of four measurements are shown in Fig. 4, two with tag ID 1 $\left(t_{\mathrm{wait}}=5 \mu \mathrm{s}\right)$ and two with tag ID $11\left(t_{\mathrm{wait}}=1005 \mu \mathrm{s}\right)$. In all four cases, the transponder is connected to the onboard unit via a $5.5 \mathrm{~m}$ long coaxial cable to exclude effects of multipath propagation, as shown in Fig. 3. Additional attenuators between the onboard unit and the transponder are used to set

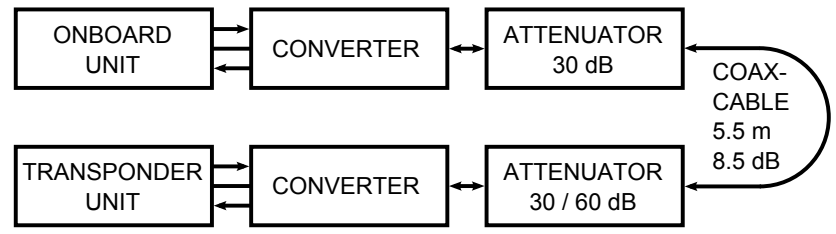

Fig. 3. Measurement setup.

a total attenuation of $68.5 \mathrm{~dB}$ in the first two and $98.5 \mathrm{~dB}$ in the second two measurements. Note that these attenuators will affect signal power in both signal directions, from the onboard unit to the transponder and back. Both units are warmed up and calibration is carried out before measurements are taken. At $68.5 \mathrm{~dB}$ attenuation, the standard deviation over 10000 samples is $3.8 \mathrm{~mm}$ for tag ID 1 and $4.9 \mathrm{~mm}$ for tag ID 11. These values show that using higher tag IDs and therefore longer waiting times in a multi-user environment will not considerably harm relative distance accuracy caused by clock phase noise. While the measurements with $68.5 \mathrm{~dB}$ one-way path loss represent real scenarios with a distance of approximately $10 \mathrm{~m}$ to the road user in a line of sight condition, measurements with $30 \mathrm{~dB}$ additional path loss are taken to model longer distances and hidden positions, where additional attenuation due to diffraction occurs. At $98.5 \mathrm{~dB}$ attenuation, the standard deviation is considerably larger due to a lower signal-to-noise ratio, but the determined standard deviations of $83.6 \mathrm{~mm}$ for tag ID 1 and $87.0 \mathrm{~mm}$ for Tag ID 11 still allow for a localization with decimeter accuracy. Again, the values for the standard deviation do not differ much between different waiting times.

\subsection{Impact of frequency offsets}

In the last section, we discussed short term variations around the mean value at static distances, but - by calibration - neglected distance offsets in our measurements. In fact, a clock frequency offset between the transponder and the onboard unit will lead to slightly different waiting times in both units, affecting the absolute measured distance. This can for example be caused by reference oscillator manufacturing tolerances, aging, or temperature drift. All these change the frequency of the oscillator slowly in time, so the frequency difference can be calculated after determining the mean distance error over a row of samples. Since the absolute timing error will be larger for longer waiting times, higher tag IDs will show greater distance offsets. To determine an example value for the offset error, the first two measurements in Fig. 4 are calibrated with the same offset value - the calibration value determined with tag ID 1 - so the mean distance error of tag ID 1 is zero. Tag ID 11 then shows a mean distance error of $\delta R=-1.457 \mathrm{~m}$, which is due to the combined clock frequency offset between the onboard unit and the transponder, accumulated over the additional waiting time of $1000 \mu$ s 

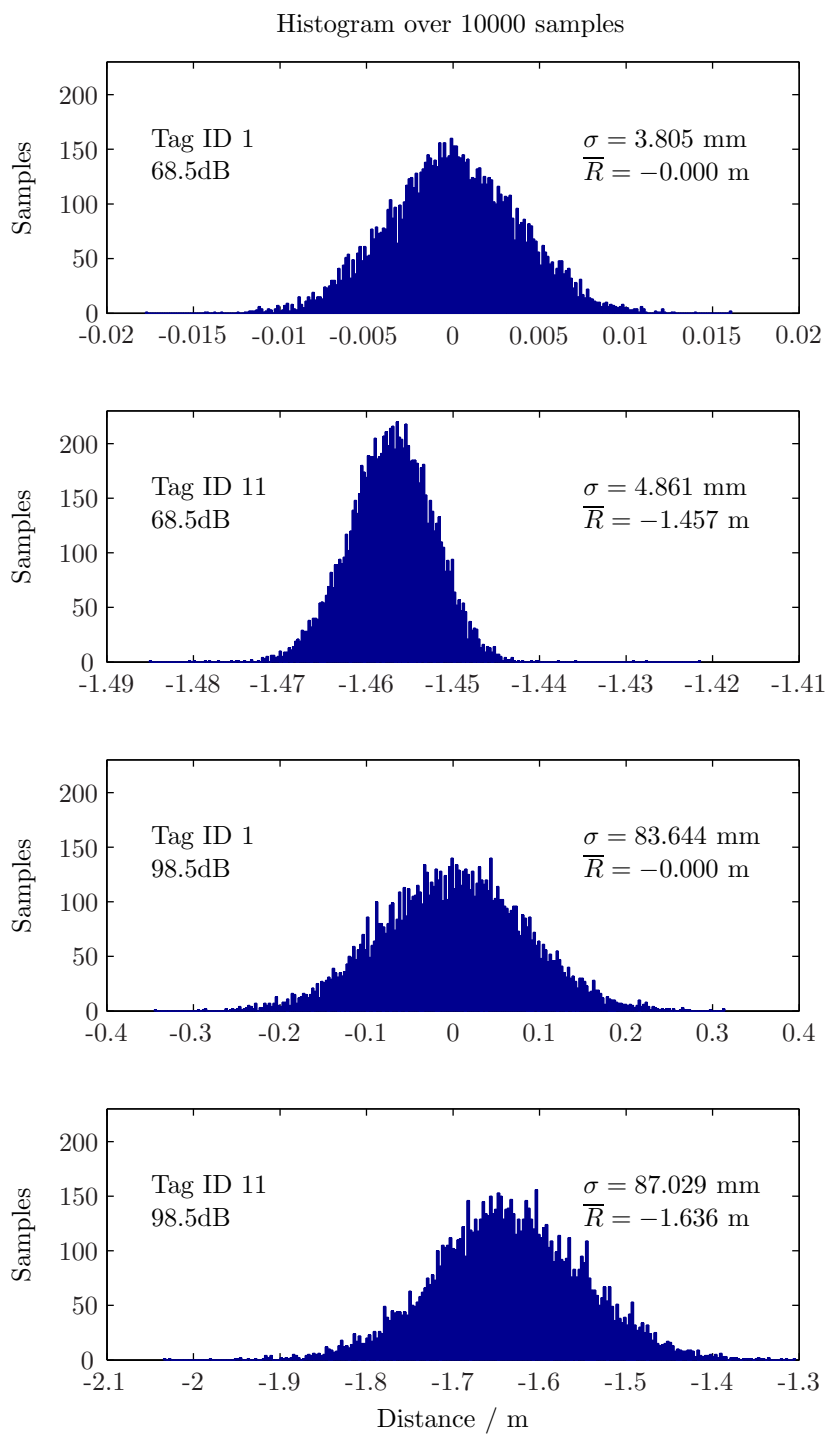

Fig. 4. Histograms for measurements with $68.5 \mathrm{~dB}$ one-way attenuation in the first and second graph, and $98.5 \mathrm{~dB}$ in the third and fourth graph. Tag ID=1 for the first and third graph, and Tag ID $=11$ for the second and fourth graph.

between timeslots 1 and 11. For this specific case, the relative clock error can be calculated to be

$\frac{\delta R \cdot 2 / c_{0}}{1000 \mu \mathrm{s}}=-9.7 \mathrm{ppm}$,

which is well within the specified range of $\pm 50 \mathrm{ppm}$ of the used crystal oscillator. To remove or minimize these errors, different approaches are possible in future systems: of course, a transponder could be recalibrated whenever the tag ID is changed. While this is possible in the current prototype stage of development, this method will not be practicable in a future system, when tag IDs are allocated dynamically in a traffic ad-hoc network via data communication. However, calibration against a "golden standard" could be carried out for every single tag ID at factory. The calibration values could be saved in a lookup table and read whenever a new tag ID was assigned. This technique would include deterministic offsets, but not temperature and aging effects. In general, the effects of clock offset and drift should therefore be reduced by using high quality reference oscillators with good temperature and aging stability, and by keeping time slots as short as possible. Using a temperature compensated crystal oscillator as a reference clock with a guaranteed accuracy of 0.5 to $1 \mathrm{ppm}$ instead of the currently used quartz oscillator could already improve the situation by a factor of 5 to 10 compared to the results presented above. Currently, Tag IDs differ in waiting time by $100 \mu$ s each, mostly due to the $65 \mu$ s long direction-of-arrival burst. In a future system, roundtrip timeof-flight and direction-of-arrival measurements will be separate and independent from each other, so the time-of-flight time slots could be reduced to a length of $10 \mu \mathrm{s}$. By combining a better oscillator with shorter timeslots, up to 100 transponders instead of 11 could be addressed with a precision ten times better than presented in the measurements above. In addition, an algorithm in the onboard unit could dynamically assign low tag IDs and therefore short waiting times to those transponders which are already close to the ego vehicle, keeping the less accurate timeslots for those which are still far away and not critical. Another option would be an on-the-fly measurement of the relative clock offset between the onboard unit and a transponder by carrying out two distance measurements in one cycle, one with a very short waiting time and one with a long one. The clock offset could then be calculated in the same way as it is calculated in the analysis above, and the determined value could be considered in the computed distance values.

\subsection{Multipath propagation}

Another effect on distance precision can be multipath propagation. Due to the limited bandwidth of the system, only multipath responses that differ by more than $\Delta R$ in path length can be distinguished by the system, while signal paths with a smaller separation cannot be resolved and will lead to a distance error due to their combination into one correlation maximum. In theory, the minimum resolvable path length separation of two signals, consisting of rectangular pulses with a duration of $\tau_{\mathrm{p}}=1 / B$ is (Klausing and Holpp, 2000)

$$
\Delta R=\frac{c}{B}
$$

which evaluates to $5 \mathrm{~m}$ for a pulse bandwidth of $60 \mathrm{MHz}$. Figure 5 shows the correlation results for 5 measurements with two signal paths, introduced by a power splitter and combiner with different cable lengths between the onboard unit and the transponder. The length of the second path is changed in 5 steps, while the system is calibrated so that the first path shows a distance of zero. It must be noted that the displayed distance for the second path is half the true value of 


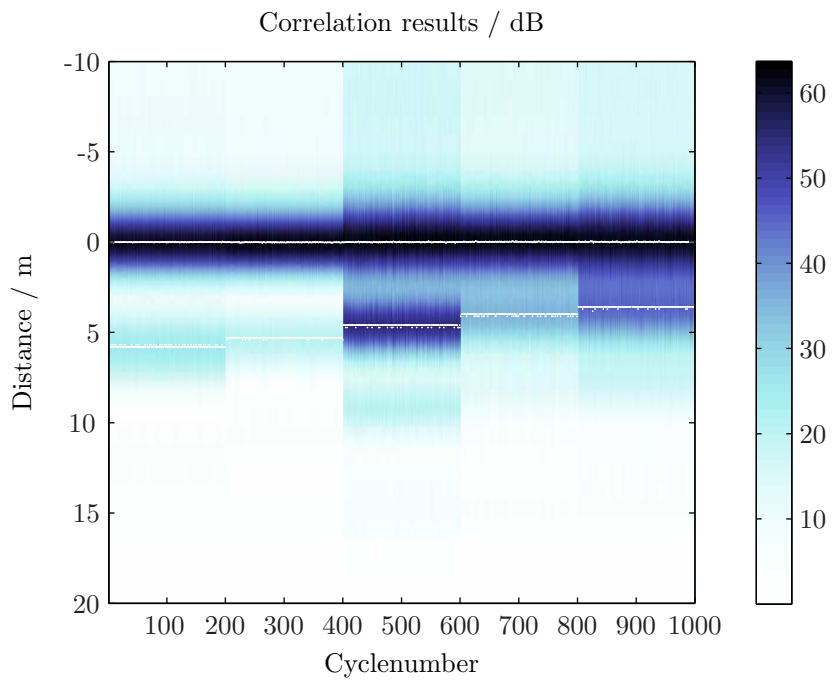

Fig. 5. Correlation results for 5 different multipath lengths, varying from 11 to $6.68 \mathrm{~m}$. The white lines show the distance output of the system for the first and second maximum. The different intensities for the multipath maxima are a result of interference between the two paths.

the multipath length, since the system calculates the distance to the transponder, which is one half of the travelled distance of the signal. The shortest path difference at which the system can still resolve the two signals is found to be at $6.68 \mathrm{~m}$. To improve the multipath resolution, superresolution algorithms could be used in future systems. According to previous research (Morhart and Biebl, 2010), these could improve the resolution of the system by a factor of approximately two. In practice, the multipath situation changes rapidly due to the movement of the ego vehicle and the transponders in traffic, while the line of sight path, if available, changes more slowly. By applying tracking algorithms in the onboard computer, some multipath situations can thus be resolved after a short time and measurement errors can be reduced.

\subsection{Outdoor measurements}

Finally, after the preceding laboratory measurements, a real world measurement shall be presented in order to show the functionality of the $5.768 \mathrm{GHz}$ system in an outdoor traffic scenario. Figure 6 depicts the correlation results as output by the onboard unit, in raw format, without any filtering or tracking applied. For the shown setup, a pedestrian starts at a distance of $10 \mathrm{~m}$ from the car which is equipped with the onboard unit. The pedestrian is then slowly moving away to a distance of $100 \mathrm{~m}$, before he comes back. At all times, the person is blocking the line of sight between the car and the transponder, including body attenuation and diffraction effects to the measurement. Even with the transponder screened by the human body, detection ranges of more than $100 \mathrm{~m}$ can easily be reached.

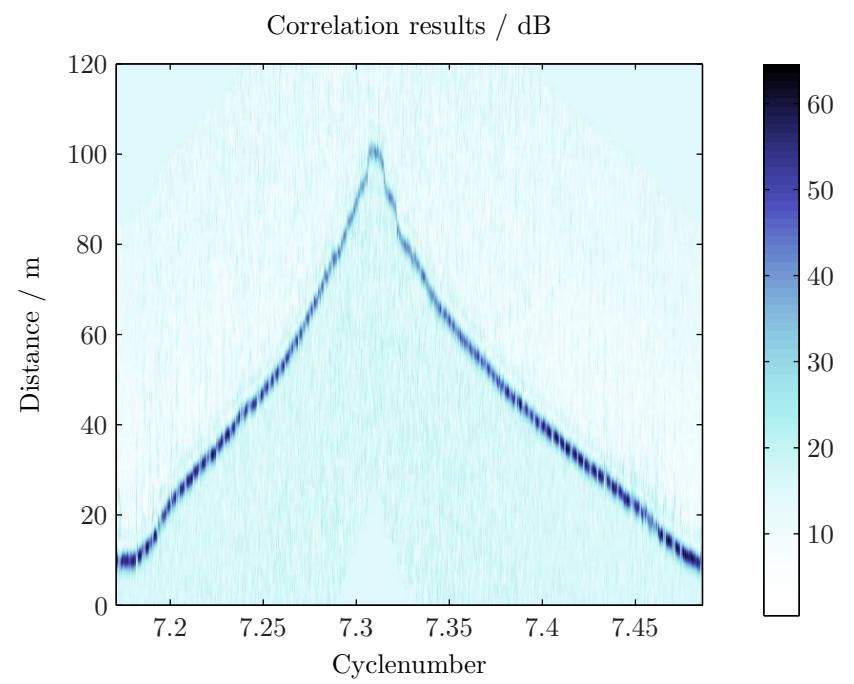

Fig. 6. Correlation results for an outdoor measurement, where the transponder is screened by the human body.

\section{Conclusions and outlook}

In the preceding sections, we presented a cooperative sensor system which is able to localize vulnerable road users in order to improve traffic safety. The system is usable at 2.44 and $5.768 \mathrm{GHz}$ to offer the ability to evaluate localization technology at automotive safety related frequency bands. In outdoor measurements, a range of more than $100 \mathrm{~m}$ can be reached, while laboratory measurements show a precision in the range of one centimeter at high signal to noise ratios and with low tag IDs. Naturally, lower signal to noise ratios will degrade accuracy, but even at a path loss of $98.5 \mathrm{~dB}$ one-way, standard deviations below ten centimeters can be observed. Using higher tag IDs, which means using longer waiting times before retransmission of the localization signal, will introduce bigger measurement errors due to clock offset and drift, but a list of countermeasures is given to minimize their impact.

The current system only supports a number of fixed timeslots, and every transponder needs to have a fixed tag ID assigned. In future systems, tag IDs will need to be allocated dynamically in order to accept upcoming users down the road into the ad-hoc network. Dynamic management of the tag IDs will then not only provide precise distance measurements to those transponders that need accurate localization because they are in a close or critical situation, but will also allow for anonymization of the transponders. This dynamic allocation mechanism requires fast and robust data communication capabilities. With the current system, these would be limited to simple modulation schemes, due to the use of hardware amplitude modulators and demodulators. In order to support modern modulation schemes for data transmission, a new system is currently being developed. With the socalled SafeTAG 2.0 system, it will be possible to use three 
channels - one for distance measurement, one for data communication and one for network management - to improve the performance of the sensor system. In this new system, a TCXO-based reference clock with a guaranteed frequency tolerance of $\pm 0.5 \mathrm{ppm}$ over temperature will be used in order to reduce offset errors in the distance measurement. Additionally, the direction-of-arrival estimation will be accomplished in the data channel, allowing shorter timeslots in the roundtrip time of flight transmission scheme, thereby reducing distance measurement errors even further.

Acknowledgement. This work results from the joint project KoTAG, which is part of the project initiative Ko-FAS, and has been funded by the German Bundesministerium für Wirtschaft und Technologie (Federal Department of Commerce and Technology) under grant number 19S9011C.

\section{References}

Electronic Communications Committee: ECC Decision of March 142008 on the harmonised use of the $5875-5925 \mathrm{MHz}$ frequency band for Intelligent Transport Systems (ITS), available at: http: //www.cept.org/ecc/deliverables, 2008.

Gandhi, T. and Trivedi, M.: Pedestrian Protection Systems: Issues, Survey, and Challenges, Intelligent Transportation Systems, IEEE Transactions on, 8, 413-430, doi:10.1109/TITS. 2007.903444, 2007.
Geissler, U., Trebing, A., Westhofen, D., and Zecha, S.: Intelligent collision risk prediction using cooperative sensor systems, Proceedings of the International Workshop on Intelligent Transportation (WIT), 8, 41-44, 2011.

Klausing, H. and Holpp, W.: Radar mir realer und synthetischer Apertur, Oldenbourg Verlag, 1st Edn., 2000.

Lüke, H. D.: Korrelationssignale, Springer Verlag, 1992.

Morhart, C. and Biebl, E. M.: Cooperative multi-user detection and ranging based on pseudo-random codes, Adv. Radio Sci., 7, 5559, doi:10.5194/ars-7-55-2009, 2009.

Morhart, C. and Biebl, E. M.: High resolution time of arrival estimation for a cooperative sensor system, Adv. Radio Sci., 8, 6166, doi:10.5194/ars-8-61-2010, 2010.

Roehr, S., Gulden, P., and Vossiek, M.: Precise Distance and Velocity Measurement for Real Time Locating in Multipath Environments Using a Frequency-Modulated Continuous-Wave Secondary Radar Approach, Microwave Theory and Techniques, IEEE Transactions on, 56, 2329-2339, doi:10.1109/TMTT.2008. 2003137, 2008.

Schwarz, D., Kloeden, H., and Rasshofer, R.: Ko-TAG - Cooperative Sensor Technology for Traffic Safety Applications, Proceedings of the International Workshop on Intelligent Transportation (WIT), 8, 31-34, 2011.

Statistisches Bundesamt Deutschland: Verkehr - Verkehrsunfälle 2010, Fachserie 8 Reihe 7, Statistisches Bundesamt Deutschland, 2010. 